\title{
Trombosis venosa portal e hiperplasia nodular regenerativa hepática; posible efecto adverso asociado a bevacizumab y oxaliplatino
}

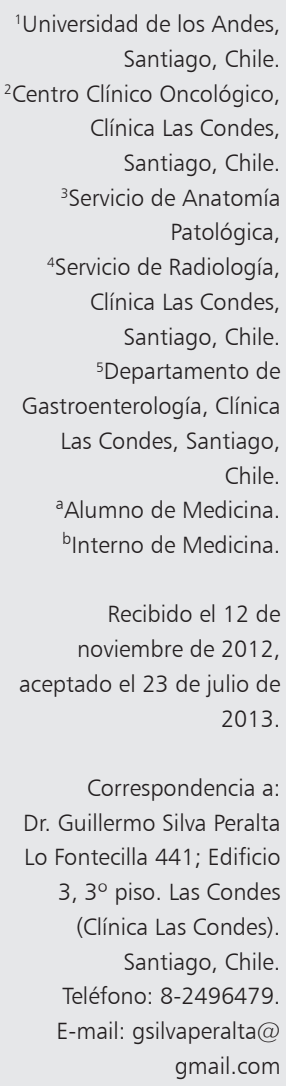

'Universidad de los Andes, Santiago, Chile. ${ }^{2}$ Centro Clínico Oncológico, Clínica Las Condes, Santiago, Chile. ${ }^{3}$ Servicio de Anatomía Patológica, ${ }^{4}$ Servicio de Radiología, Clínica Las Condes, Santiago, Chile.

${ }^{5}$ Departamento de Gastroenterología, Clínica Las Condes, Santiago, Chile.

aAlumno de Medicina. bInterno de Medicina.

Recibido el 12 de noviembre de 2012, aceptado el 23 de julio de 2013.

Correspondencia a: Dr. Guillermo Silva Peralta Lo Fontecilla 441; Edificio 3, $3^{\circ}$ piso. Las Condes (Clínica Las Condes). Santiago, Chile. Teléfono: 8-2496479. E-mail: gsilvaperalta@ gmail.com

\author{
DANIELA FLUXÁ C..$^{1, a}$, SEBASTIÁN SALAS M. ${ }^{1, b}$, CARLOS REGONESI M.², \\ LUIS CONTRERAS M. ${ }^{3}$, ALEX WASH F. ${ }^{4}$, GUILLERMO SILVA P. ${ }^{5}$
}

\section{Portal vein thrombosis and nodular regenerative hyperplasia associated with the use of bevacizumab and oxaliplatin. Report of one case}

Nodular regenerative hyperplasia (NRH) consists in diffuse transformation of the hepatic parenchyma into small regenerative nodules without fibrosis, secondary to vascular occlusion and flow alterations. This gives a nodular appearance to the liver, as there is atrophy and compensatory hypertrophy of hepatocytes. We report a 69-year-old male who suffered of colon cancer and was treated with Oxaliplatin $(O X)$ and Bevacizumab (B). During treatment with B the patient presented a partial thrombosis of the portal vein, that one year later became permeable. Esophageal varices were found in an upper digestive endoscopy. Hepatic tests were normal. A liver biopsy was performed and informed nodular regenerative hyperplasia. Thus, the different factors that could explain this pathology are analyzed. $B$, a monoclonal antibody against vascular endothelial growth factor, reduces the anti-apoptotic, anti-inflammatory and survival effects produced by this factor, affecting the vascular protection of the endothelial cell. On the other hand, OX activates metalloproteinases and depletes sinusoidal glutathione producing sinusoidal lesions. Thus, (OX) would be associated with sinusoidal obstruction and NRH sporadically. It is important to discuss the possible etiologic factors that can cause NRH reviewing the hepatotoxic effects caused by both drugs.

(Rev Med Chile 2013; 141: 1344-1348).

Key words: Antibodies, monoclonal, humanized; Bevacizumab; Focal nodular hyperplasia; Hypertension, portal; Organometallic compounds; Oxaliplatin.
L a hipertensión portal no cirrótica (HPNC) se caracteriza con mayor frecuencia por un aumento de la resistencia al flujo portal, a nivel prehepático, intrahepático o posthepático ${ }^{1-3}$. Las causas prehepáticas se deberían en su mayoría a trombosis de la vena porta, de sus ramas o peri sinusoidales ${ }^{1,2}$. Secundario a esto último se producirían cambios en la arquitectura hepática, consistentes en transformación nodular, conocida como hiperplasia nodular regenerativa $(\mathrm{HNR})^{1,2}$. La HNR se caracteriza por transformación difusa del parénquima hepático en pequeños nódulos re- generativos con ausencia o mínima fibrosis ${ }^{4,5}$. Esta patología puede ser secundaria a tóxicos, fármacos, trastornos reumatológicos y hemato-oncológicos, los que estarían asociados a alteraciones sistémicas, tales como artritis reumatoide, poliarteritis nodosa, lupus eritematoso sistémico, policitemia vera, linfoma de Hodgkin y no Hodgkin, y drogas $^{4}$. A pesar de que existen diversos factores que causan HNR, esta es una condición infrecuente, generalmente asintomática que se descubre incidentalmente ${ }^{6}$, motivo por el cual se presenta el caso clínico y se discute su eventual patogenia. 
Hipertensión portal e hiperplasia nodular regenerativa - D. Fluxá et al

\section{Caso clínico}

Paciente de sexo masculino de 69 años, con antecedentes de cáncer de colon y metástasis hepáticas (MH) el año 2004. Fue sometido a cirugía, realizándose hemicolectomía derecha, resección y radiofrecuencia de lesiones hepáticas. La biopsia intraoperatoria analizó fragmentos hepáticos que describen $\mathrm{MH}$. Tres semanas después de la cirugía el paciente inicia quimioterapia consistente en: cetuximab, oxaliplatino (OX), 5 flurouracilo (5FU) y leucovorina (LV) cada dos semanas. Debido a la evolución favorable del paciente se retira el OX y la LV al sexto ciclo de quimioterapia. A los dos meses se objetiva nueva $\mathrm{MH}$ por lo que se retoma el OX bimensual y tras seis ciclos el paciente es sometido a lobectomía hepática izquierda. El examen microscópico de la biopsia informó que el resto del parénquima hepático presentaba arquitectura conservada. Debido a la recidiva se cambia el esquema por $5 \mathrm{FU}$, irinotecan (I) y bevacizumab
(B), el cual fue administrado cada dos semanas. $\mathrm{Al}$ décimo ciclo se eliminó el I de la terapia por buena respuesta y se continuó con los dos agentes restantes. Tras once ciclos de quimioterapia, el paciente presentó trombosis parcial de la vena porta (TPVP) en tomografía axial computada (TAC) (Figura 1A). Tras anticoagulación oral con acenocumarol por cinco meses, el paciente reinició quimioterapia con $\mathrm{B}$, realizándose diecinueve ciclos más.

El año 2008 se pesquisaron várices esofágicas grado 2 de la clasificación de Baveno, mediante endoscopia digestiva alta $^{7}$. En TAC, se constató desarrollo de circulación colateral porto-sistémica, con repermeabilización de la vena umbilical y múltiples colaterales en hilio esplénico, perigástricas y periesofágicas, con repermeabilización prácticamente completa de la vena porta con flujo presente (Figura 1B). Las pruebas hepáticas fueron normales. A raíz de ello se realizó biopsia hepática para estudio etiológico. La biopsia informó HNR (Figura 2).
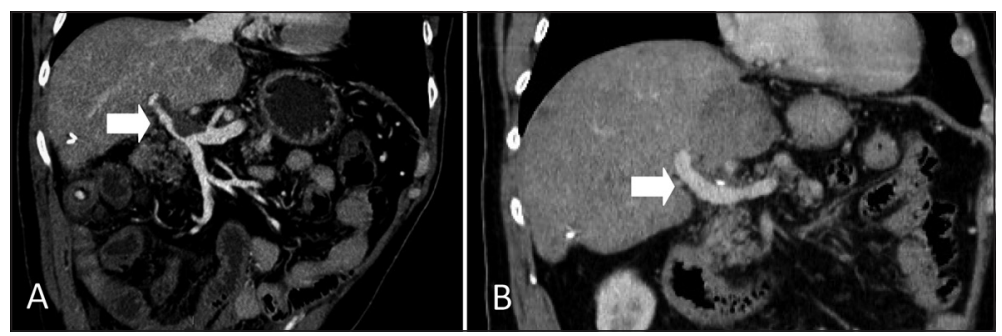

Figura 1. A. Tomografía axial computada (TAC): Se señala trombosis parcial de la vena porta $(\rightarrow)$. En la figura $\mathbf{B}$ : se aprecia repermeabilización de la vena porta $(\rightarrow)$.

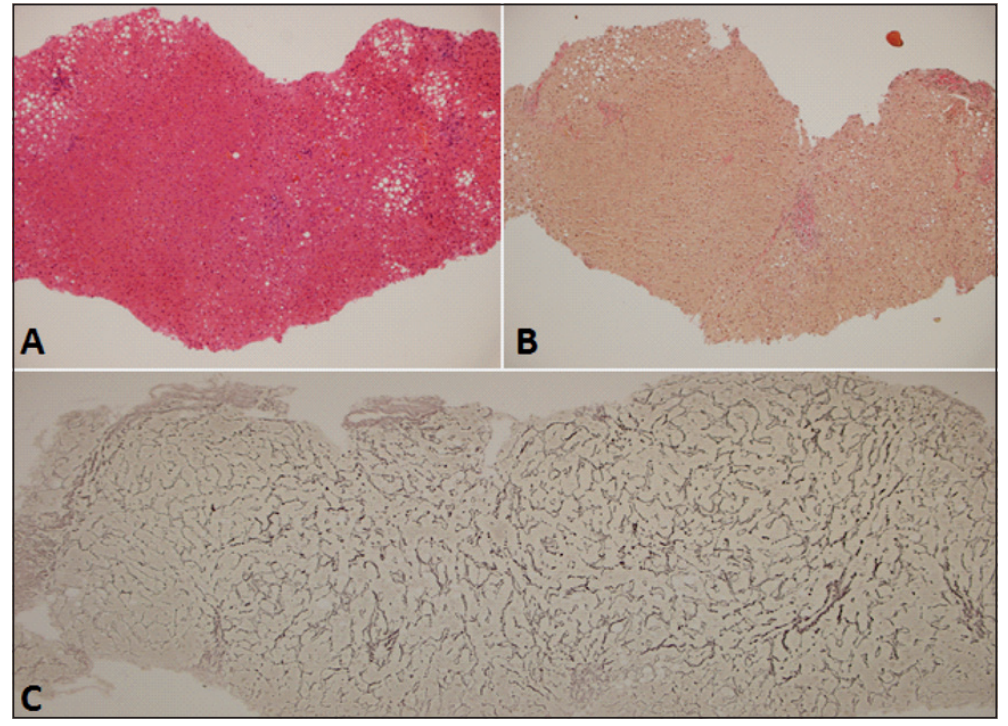

Figura 2. Biopsia Hepática. Figura (A) Hematoxilina-Eosina (100X), Figura (B) tinción Van Gieson (100X), en ambas leve esteatosis macrovacuolar sin bandas fibrosas. Figura (C) tinción de retículo (100X) en que se evidencia claramente hiperplasia nodular regenerativa (HNR). 


\section{Discusión}

En la HNR el tamaño de los nódulos es menor a tres milímetros aunque se han descrito nódulos mayores, frecuentemente compuestos por múltiples nódulos pequeños ${ }^{4}$. El patrón nodular de la HNR surgiría como consecuencia de una alteración en el flujo sanguíneo ${ }^{4,6}$, en donde se observaría frecuentemente anormalidades en las venas portales y/o centrales ${ }^{4}$. El trastorno vascular produce atrofia de los hepatocitos en la zona isquémica e hipertrofia compensatoria en aquellas donde la vascularización no está afectada, otorgando al hígado el aspecto nodular difuso ${ }^{8}$.

La HNR suele permanecer asintomática durante años. Las pruebas hepáticas generalmente son normales, sólo en $25 \%$ de los pacientes hay aumento de las fosfatasas alcalinas ${ }^{4,5}$. La enfermedad tiene un curso benigno, a pesar de reportarse hipertensión portal (HP) en 50\% de los pacientes ${ }^{8}$ y ser ésta y sus complicaciones las que dominan la presentación clínica y el curso de la enfermedad ${ }^{4}$. En la HNR la función hepática está conservada e infrecuentemente se observa ascitis o encefalopatía hepática ${ }^{8,9}$. El desarrollo de la enfermedad tiene un curso lentamente progresivo $^{8}$.

El diagnóstico diferencial de la HNR debe hacerse con adenoma hepático $(\mathrm{AH})$, hiperplasia focal nodular (HNF), transformación nodular parcial (TNP), nódulos de regeneración grande (NRG), y la cirrosis completa e incompleta ${ }^{4}$. Las patologías antes mencionadas pueden diferenciarse entre sí según sus características. En la HNR hay una distribución nodular numerosa y difusa, distinta a la HNF y el $\mathrm{AH}$, los que usualmente presentan un nódulo único ${ }^{4}$. En cuanto a la TNP y los NRG, presentan distribución nodular tanto única como múltiple, siendo los nódulos mayores a tres centímetros ${ }^{4}$. Por último, podemos distinguir la HNR de la cirrosis por la presencia de fibrosis en esta última, la cual está ausente en la HNR $\mathrm{HN}^{4}$. Es importante destacar que el diagnóstico de HNR se hace en base a histología ${ }^{4}$.

Este caso clínico hace mención al uso de $B$, anticuerpo monoclonal humanizado utilizado como quimioterapia en el cáncer colorrectal metastásico. Éste actuaría inhibiendo la angiogénesis al unirse a una secuencia específica del factor de crecimiento endotelial vascular (VEGF), impidiendo la unión de todas las isoformas del VEGF con sus receptores. Esto aumentaría el riesgo de trombosis arterial al alterar la célula endotelial, la que juega un rol esencial en la homeostasis vascular $^{10,11}$. Además, el VEGF tiene efectos antiapoptóticos, antiinflamatorios y de supervivencia, protegiendo al endotelio vascular ${ }^{10,11}$. También el VEGF aumenta la producción de óxido nítrico (NO) y prostaglandina I2 (PGI2) a través de etapas intermedias que involucran la producción de óxido nítrico sintetasa endotelial (eNOS) y de ciclooxigenasa 1 (COX-1) respectivamente. De esta forma la disminución del VEGF produciría una disminución de NO y PGI2, ocasionando vasoconstricción y aumento en la agregación y adhesión plaquetaria al endotelio vascular ${ }^{10-12}$. Es así como un inhibidor del VEGF como el B puede alterar la función del endotelio y causar defectos en la pared vascular, exponiendo fosfolípidos protrombóticos en la membrana plasmática luminal y matriz subyacente, produciendo accidentes de placa, activación plaquetaria, formación in situ de trombos y trombosis arterial ${ }^{10,12}$. Lo anterior se refiere sobre todo al daño arterial, sin embargo, se postula que un mecanismo similar ocurriría en trombosis venosa ${ }^{12}$. Por otro lado, se demostró en modelos experimentales que el $\mathrm{B}$ puede inducir agregación plaquetaria, degranulación y trombosis a través de la formación de complejos con el VEGF y activación del receptor Fc gamma RIIa IgG de la plaqueta ${ }^{10,12}$. Por lo tanto, la HNR y eventos trombóticos del paciente podrían ser consecuencia de efectos adversos del B, como resultado de la oclusión de ramas de la vena porta intrahepática. Sin embargo, otros agentes quimioterapéuticos recibidos por el paciente, podrían relacionarse con la $\mathrm{HNR}$, como el OX, descrito en estudios recientes ${ }^{13,14}$. Así, el OX tendría un rol en la génesis de esta patología mediante la activación de metaloproteinasas y depleción de la glutatión sinusoidal, lo que produciría lesiones sinusoidales (dilatación, congestión y obstrucción $)^{5}$. Debido a esto el OX se ha visto asociado a obstrucción sinusoidal y a HNR cuya frecuencia oscilaría desde casos esporádicos hasta $15 \%-58 \%$ de los pacientes ${ }^{5,13}$. Además se ha sugerido un eventual efecto protector del B en el desarrollo de toxicidad vascular por OX, al regular la activación de metaloproteinasas ${ }^{14,15}$. Hay modelos experimentales que han estudiado el síndrome de obstrucción sinusoidal, en donde 
la activación de la metaloproteinasa 2 de la matriz (MMP-2) y metaloproteinasa 9 de la matriz (MMP-9) jugarían un rol mayor en la degradación de la matriz perisinusoidal y participarían en la dilatación sinusoidal ${ }^{14}$. El rol del VEGF en el síndrome de obstrucción sinusoidal, sería que éste induce la expresión de MMP-9 por la célula endotelial sinusoidal ${ }^{14}$. Entonces el bloqueo del VEGF por el B produciría regulación de la expresión de MMP-9 lo posiblemente atenuaría las lesiones sinusoidales ${ }^{14,16}$. Por lo tanto, las lesiones relacionadas con el OX serían menos frecuentes $y$ severas en pacientes tratados con $\mathrm{B}^{14,16}$. De esta forma nos parece adecuado considerar la eventual toxicidad hepática de ambos fármacos, que hace recomendable evaluarlo en el seguimiento de pacientes sometidos a quimioterapia con estos fármacos.

\section{En conclusión}

Dentro de los factores señalados, es posible que el bevacizumab tenga relación con la HNR a través de daño endotelial. Este mecanismo podría ser la causa tanto de la trombosis parcial de la vena porta, como de sus ramas intrahepáticas, causando la HNR. También es planteable que el oxaliplatino pueda explicar esta patología dado que tiene un rol importante en la producción de lesiones sinusoidales que podrían ocasionar HNR. De ser el oxaliplatino como la causa de la HNR, el uso prolongado de $\mathrm{B}$ posterior al de oxaliplatino no habría actuado como factor protector de estas lesiones. Finalmente, los mecanismos de producción de HNR en este caso no están del todo aclarados, ya que en este paciente hay dos drogas potencialmente hepatotóxicas. Sin embargo, nos parece de importancia destacar los efectos laterales teóricos de estos fármacos de modo que estemos atentos a esta eventual complicación.

\section{Referencias}

1. Sarin SK, Kapoor D. Non-cirrhotic portal fibrosis: Current concepts and management. J Gastroenterol Hepatol 2002; 17 (5): 526-34.

2. Hillaire S, Bonte E, Denninger MH, Casadevall N, Cadranel JF, Lebrec D, et al. Idiopathic non-cirrhotic intrahepatic portal hypertension in the West: a re-evaluation in 28 patients. Gut 2002; 51 (2): 275-80.

3. Krasinskas AM, Eghtesad B, Kamath PS, Demetris AJ,
Abraham SC. Liver transplantation for severe intrahepatic non cirrhotic portal hypertension. Liver Transpl 2005; 11 (6): 627-34.

4. Reshamwala PA, Kleiner DE, Heller T. Nodular regenerative hyperplasia: not all nodules are created equal. Hepatology 2006; 44 (1): 7-14.

5. Van den Broek M, Olde S, Driessen A, Dejong C, Bemelmans M. Nodular regenerative hyperplasia secondary to neoadjuvant chemotherapy for colorectal liver metastasis. Case report med. 2009, (2009). Disponible en http:// www.ncbi.nlm.nih.gov/pmc/articles/PMC2787019/ [Consultado el 20 de marzo de 2012].

6. Wang HM, Lo GH, Hsu PI, Lin CK, Chan HH, Chen WC, et al. Nodular regenerative hyperplasia of the Liver. J Chin Med Assoc 2008; 71 (10): 523-7.

7. De Franchis R, Pascal JP, Ancona E, Burroughs AK, Henderson M, Fleig W, et al. Definitions, methodology and therapeutic strategies in portal hypertension. A Consensus Development Workshop, Baveno, Lake Maggiore, Italy, April 5 and 6, 1990. J Hepatol 1992; 15 (1-2): 256-61.

8. Al-Mukhaizeem KA, Rosenberg A, Sherker AH. Nodular regenerative hyperplasia of the liver: an under-recognized cause of portal hypertension in hematological disorders. Am J Hematol 2004; 75 (4): 225-30.

9. Seoane A, Galeras JA, Coll S, Solà R, Bory F. Ascites as the sole clinical manifestation in a patient with nodular regenerative hiperplasia. Gastroenterol Hepatol 2003; 26 (5): 297-9.

10. Schutz FA, Je Y, Azzi GR, Nguyen PL, Choueiri TK. Bevacizumab increases the risk of arterial ischemia: a large study in cancer patients with a focus on different subgroup outcomes. Ann Oncol 2011; 22 (6): 1404-12.

11. Ferroni P, Formica V, Roselli M, Guadagni F. Thromboembolic events in patients treated with antiangiogenic drugs. Curr Vasc Pharmacol 2010; 8 (1): 102-13.

12. Ranpura V, Hapani S, Chuang J, Wu S. Risk of cardiac ischemia and arterial thromboembolic events with the angiogenesis inhibitor bevacizumab in cancer patients: a meta-analysis of randomized controlled trials. Acta Oncol 2010; 49 (3): 287-97.

13. Béchade D, Désolneux G, Fonck M, Soubeyran I, Bécouarn Y, Evrard S. Regenerative nodular hyperplasia of the liver related to oxaliplatin-based chemotherapy. Presse Med 2013; 42 (1): 102-7.

14. Rubbia-Brandt L, Lauwers GY, Wang H, Majno PE, Tanabe K, Zhu AX, et al. Sinusoidal obstruction syndrome and nodular regenerative hyperplasia are frequent oxaliplatin-associated liver lesions and 
partially prevented by bevacizumab in patients with hepatic colorectal metastasis. Histopathology 2010, 56 (4): 430-9.

15. Wicherts DA, de Haas RJ, Sebagh M, Ciacio O, Lévi F, Paule B, et al. Regenerative nodular hyperplasia of the liver related to chemotherapy: impact on outcome of liver surgery for colorectal metastases. Ann Surg Oncol
2011; 18 (3): 659-69.

16. Ribero D, Wang H, Donadon M, Zorzi D, Thomas $\mathrm{MB}$, Eng $\mathrm{C}$, et al. Bevacizumab improves pathologic response and protects against hepatic injury in patients treated with oxaliplatin-based chemotherapy for colorectal liver metastases. Cancer 2007; 110 (12): 2761-7. 